Rev. Roum. Chim.,
ACADEMIA ROMÂNĂ
Revue Roumaine de Chimie
http://web.icf.ro/rrch/

\title{
INFLUENCE OF THE INJECTED SAMPLE COMPOSITION ON THE RETENTION PARAMETERS OF POLAR COMPOUNDS UNDER ZIC-HILIC MECHANISM IN LIQUID CHROMATOGRAPHY
}

\author{
Maria TANASE and Victor DAVID* \\ University of Bucharest, Faculty of Chemistry, Department of Analytical Chemistry, Sos. Panduri, no 90, \\ Bucharest 050663, Roumania
}

Received May 30, 2019

\begin{abstract}
This study is focused on the influence of the sample composition and volume on the retention of two tested compounds (glycyl-Lleucine and glycyl-L-alanine) under zwiterionic hydrophilic liquid chromatography mechanism (Zic-Hilic), using a column loaded with zwitterionic sulfobetaine covalently attached to porous silica as stationary phase. A standard mobile phase composition was used $(80 \%$ acetonitrile and $20 \%$ aqueous component with various contents of ammonium acetate) for investigating the possible retention modifications of studied compounds (retention time and peak shape) when sample diluent was identical or different to the composition of the mobile phase. It was shown that small

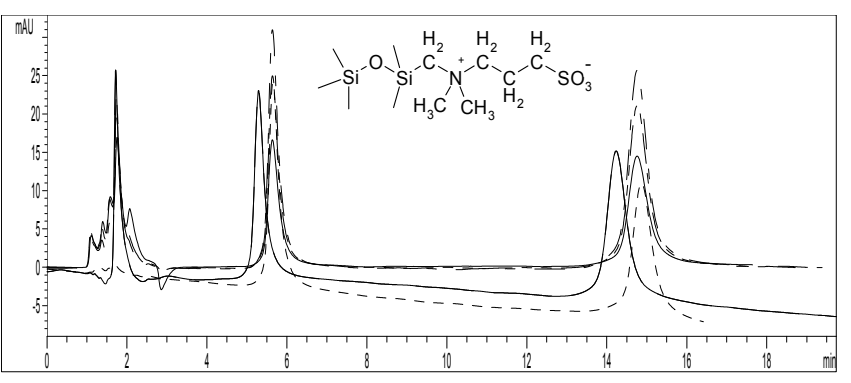
variations in the composition of the injected solvent do not affect significantly the retention parameters (retention factor, peak efficiency and simmetry) of the tested compounds, when the injected volume was at most $20 \mu \mathrm{L}$. Higher injected volumes $(50 \mu \mathrm{L})$ can influence the retention of the less retained compound, glycyl-L-leucine, while the more retained compound, glycyl-L-alanine, is not influenced by the modification of the concentration of ammonium salt in sample diluent.
\end{abstract}

\section{INTRODUCTION}

Injection volume is a major experimental parameter in liquid chromatography (LC) influencing the sensitivity of the analytical method for quantitative purposes. ${ }^{1}$ High injection volumes in reversed-phase LC are possible, with several conditions for the sample diluent: $i$ ) injection diluent is identical or very close as the composition of the mobile phase $\left.{ }^{2-4} i i\right)$ injection diluent is hydrophobic and retained in the stationary phase after injection step. ${ }^{5-9}$ The volume and composition of the injection solvent can influence the peak shape in hydrophilic liquid chromatography (Hilic) mechanism. ${ }^{10}$ Organic solvents, such as acetonitrile, ethanol, methanol, i-propanol, or dimethylsulfoxide can be succesfully used as injection solvent, but the study reporting this possibility has been focused on small injection volume. $^{11}$

The retention zwiterionic hydrophilic liquid chromatography (Zic-Hilic) is influenced by a set of experimental parameters, such as mobile phase composition, ${ }^{12-15}$ or temperature. ${ }^{16,17}$ In case of Zic-Hilic mechanism the composition of sample diluent and the injection volume have been rarely debated. Taking into consideration the importance of this retention mechanism in current applications, ${ }^{18}$ and the complexity of the retention process under this mechanism, ${ }^{19}$ two injection parameters (injection volume and sample composition) were studied for two target analytes by using a typical stationary phase in this

\footnotetext{
${ }^{*}$ Corresponding author: victor.david@chimie.unibuc.ro
} 
mechanism (zwitterionic sulfobetaine chemically bonded to the silica surface) ${ }^{20}$

\section{EXPERIMENTAL}

This study was performed on an Agilent 1100 Series liquid chromatograph (Agilent Technologies) in the following configuration: degasser (G1379A), binary pump (G1312A), auto sampler (G1313A), column thermostat (G1316A), and diode array detector (G1315A). Chromatographic data were acquired by means of Agilent Chemstation software rev. B.01.03.

All solvents were HPLC (gradient) grade. Acetonitrile and methanol were purchased from Sigma-Aldrich (Germany). Reagent (ammonium acetate) was pro-analysis grade purchased from Merck (Germany). Water (resistivity minimum $18.2 \mathrm{M} \Omega$ and TOC maximum $30 \mathrm{ppb}$ ) was produced within the laboratory with a TKA Lab HP 6UV/UF instrument.

A SeQuant Zic-HILIC (Merck) stationary phase containing a zwitterionic sulfobetaine covalently attached to porous silica (with structure depicted in Fig. 1), accordingly to producer guide, packed into a column with $150 \mathrm{~mm}$ length and $4.6 \mathrm{~mm}$ i.d., and $5 \mu \mathrm{m}$ particle size was used under isocratic elution conditions. All experiments were performed using a flow-rate of $1 \mathrm{~mL} / \mathrm{min}$. UV detection was used by monitoring the absorbance at 205, 215 and $220 \mathrm{~nm}$ wavelength, depending on the analyte property of absorbing in UV range. Injection volume was set up to various values, specified in the content of this paper. Column temperature was set up to $25^{\circ} \mathrm{C}$.<smiles>C[N+](C)(CCC[Se-])C[Si](C)(C)O[Si](C)(C)C</smiles>

Fig. 1 - Chemical structure of zwitterionic sulfobetaine covalently attached to porous silica.

Tested compounds in this study were glycyl-L-leucine and glycyl-L-alanine. Stock solutions of these compounds with concentration of $500 \mu \mathrm{g} / \mathrm{mL}$ were prepared using different sample solvents indicated in this study. These stock solutions were diluted with sample diluent, accordingly to the injection volume, such that the amount of analyte loaded into the column to be about the same, i.e. $500 \mathrm{ng}$.

The values of retention factor $(k)$ were calculated with the formula $k=\left(t_{R}-t_{0}\right) / t_{0}$, where $t_{\mathrm{R}}$ is the absolute retention time of studied analyte, and $t_{0}$ is the dead time indicator, measured by the retention time of toluene.

\section{RESULTS AND DISCUSSION}

Generally, the sample diluent injected into the chromatographic column has to be identical or very similar to the mobile phase. Other solvents miscible with the mobile phase can be used, but their volume should be as small as possible (1 $5 \mu \mathrm{L}$ ). On the other hand, as a result of their high polarity, these compounds are not soluble in acetonitrile or methanol, and therefore injections of standard solutions with these diluents are not possible. Therefore, this study was focused on the injected samples based on aqueous diluents with composition similar to the mobile phase used for the chromatographic elution process.

The mobile phase in Zic-Hilic liquid chromatography consists of acetonitrile and aqueous component containing small concentration of a salt (the most recommended salt being ammonium acetate). ${ }^{21-23}$ In this study, various salt concentrations of the aqueous component of the mobile phase was used while the content of acetonitrile was kept constant to a value of $80 \%$ (volume/volume). The role of the added salt to the mobile phase is to supress the strong interactions of injected analyte, ${ }^{24}$ which can be charged as anion, cation or zwitterion, with the electrostatic moieties from stationary phase (denoted in the next model by $\mathrm{A}^{+}$, which is the quaternary ammonium group, and $\mathrm{B}^{-}$, which is the sulphonate group), as a result of the next two competitive equilibria:

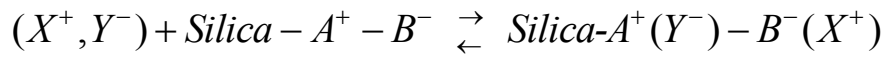

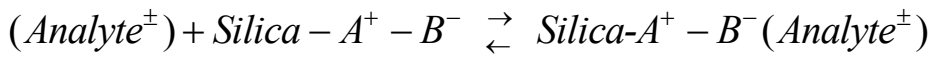

These two competitive equilibria take place during the elution process, and within the band (zone) resulted from sample injection into the column. Identical composition of the injected sample with the mobile phase does not influence the partition equilibrium of the charged analyte between mobile and stationary phase. ${ }^{25}$ HPLC experiments carried out on mobile phase containing, for example, $80 \%$ acetonitrile and $20 \%$ aqueous component containing $2.510^{-3} \mathrm{moles} / \mathrm{L}$ ammonium acetate, showed that the main retention parameters are not significantly modified by the increase of the injected sample volume, as can be seen from Fig. 2, where their variation are illustrated for the two studied compounds. The corresponding overlaid chromatograms are depicted in Fig. 3, where the average retention time values for the two studied compounds are indicated. As can be seen from the three graphs included in Fig. 2, almost all retention parameters 
did not vary significantly with the increase of the injected sample volume. Only the simmetry of the peak of glycyl-L-leucine was improved by injecting $20 \mu \mathrm{L}$ volume of sampe containing this dipeptide. Similar results were obtained for mobile phase containing $80 \%$ acetonitrile and $20 \%$ aqueous component containing $5 \cdot 10^{-3}, 10 \cdot 10^{-3}$, $15 \cdot 10^{-3}, 20 \cdot 10^{-3}$ and $25 \cdot 10^{-3}$ moles $/ \mathrm{L}$ ammonium acetate. The variations of the chromatographic parameters for the two studied compounds, expressed as relative standard deviation (\%) are given in Table 1.

As a conclusion, injections of moderate volumes of samples containing identical sample diluent with the mobile phase have no significant influence on the retention parameters of studied compounds.

The influence of the ammonium acetate content in sample solvent was studied for the mobile phase containing $80 \%$ acetonitrile and $20 \%$ aqueous component with $20 \quad 10^{-3}$ moles/L ammonium acetate. For this purpose, the content of acetonitrile was kept as a constant value $(80 \%)$, while the concentration of ammonium acetate in aqueous component was varied from $2.510^{-3}$ to $2010^{-3}$ moles/L. The variation of the decimal logarithm of the retention factor of the two studied dipeptides on the injection volume containing as diluent various concentration of ammonium acetate in aqueous component are illustrated in Fig. 4.

For higher injection volumes the influence of the concentration of salt in sample diluent on the retention process is significant only for the less retained solute (glycyl-L-leucine). Thus, a negative peak can be noticed in the retention domain of the first eluted compound, glycyl-L-leucine (Fig. 5), while the chromatographic characteristics of the second eluting compound, glycyl-L-alanine, remained almost unchanged. The same efects were observed when the concentration of ammonium acetate in sample solvent was changed to 5 , or $2.5 \mathrm{mmoles} / \mathrm{L}$, while the concentration of ammonium acetate in mobile phase has been kept constant to $20 \mathrm{mmoles} / \mathrm{L}$.
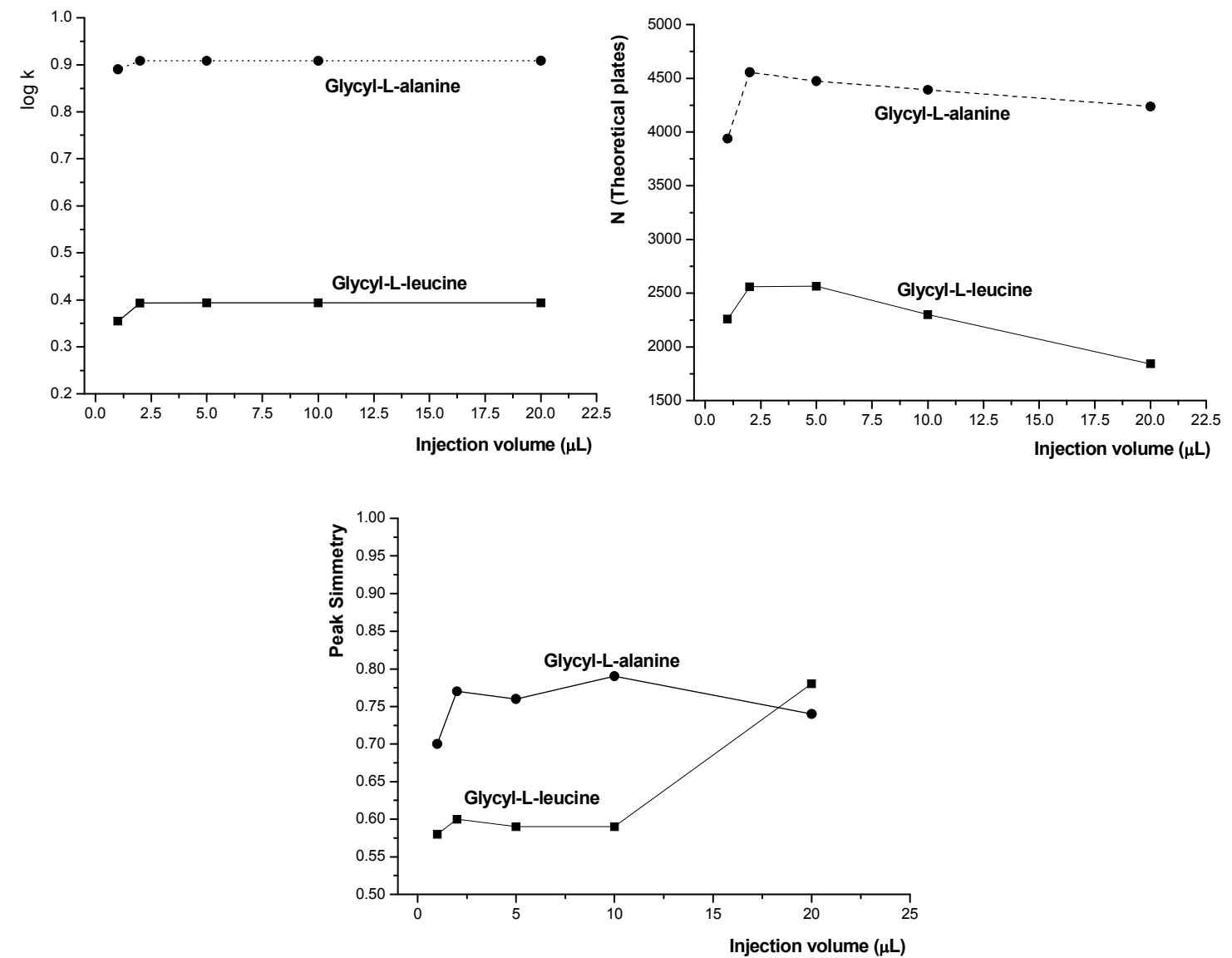

Fig. 2 - Variation of the retention factor $(k)$, peak efficiency $(N)$ and peak simmetry with the increase of injected sample volume for glycyl-L-alanine and glycyl-L-leucine, containing $80 \%$ acetonitrile and aqueous solution with $2.510^{-3} \mathrm{moles} / \mathrm{L}$ ammonium acetate. 


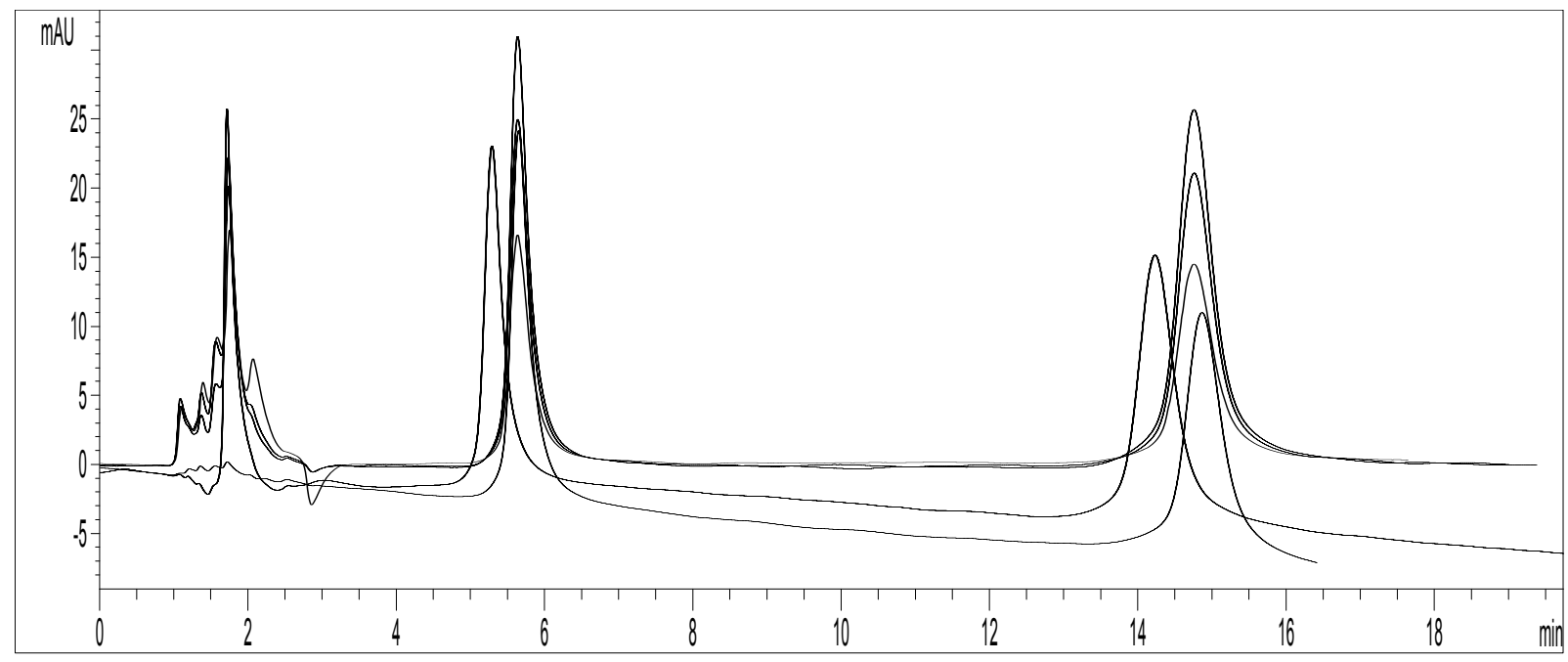

Fig. 3 - Overlaid chromatograms resulted from the injection of different sample volumes containing glycyl-L-leucine (first eluted compound at average retention time of $5.566 \mathrm{~min}$, with a relative standard deviation, RSD\%, 2.73\%) and glycyl-L-alanine (the second eluted compound at average retention time of $14.656 \mathrm{~min}$, with an $\mathrm{RSD}=1.61 \%$ ); mobile phase composition: $80 \%$ acetonitrile and $20 \%$ aqueous with $2.510^{-3} \mathrm{moles} / \mathrm{L}$.

\section{Table 1}

Values of the relative standard deviation (RSD\%) for studied compounds

of the data set obtained for injected sample volumes of $1 ; 2 ; 5 ; 10$ and $20 \mu \mathrm{L}$, with various contents of ammonium acetate

\begin{tabular}{c|c|c|c|c}
\hline $\begin{array}{c}\text { Ammonium acetate } \\
\text { concentration (moles/L) }\end{array}$ & $\begin{array}{c}\mathrm{RSD} \% \text { for } \mathrm{t}_{\mathrm{r}} \text { of } \\
\text { glycyl-L-leucine }\end{array}$ & $\begin{array}{c}\mathrm{RSD} \% \text { for } \mathrm{t}_{\mathrm{r}} \text { of } \\
\text { glycyl-L-alanine }\end{array}$ & $\begin{array}{c}\text { RSD\% for } N \text { of } \\
\text { glycyl-L-leucine }\end{array}$ & $\begin{array}{c}\mathrm{RSD} \% \text { for } N \text { of } \\
\text { glycyl-L-alanine }\end{array}$ \\
\hline $5 \cdot 10^{-3}$ & 0.11 & 0.05 & 8.42 & 5.04 \\
$10 \cdot 10^{-3}$ & 0.99 & 1.39 & 21.27 & 5.44 \\
$15 \cdot 10^{-3}$ & 0.10 & 0.09 & 3.42 & 4.26 \\
$20 \cdot 10^{-3}$ & 0.07 & 0.12 & 12.50 & 5.07 \\
$25 \cdot 10^{-3}$ & 1.3 & 2.12 & 17.81 & 16.18 \\
\hline
\end{tabular}
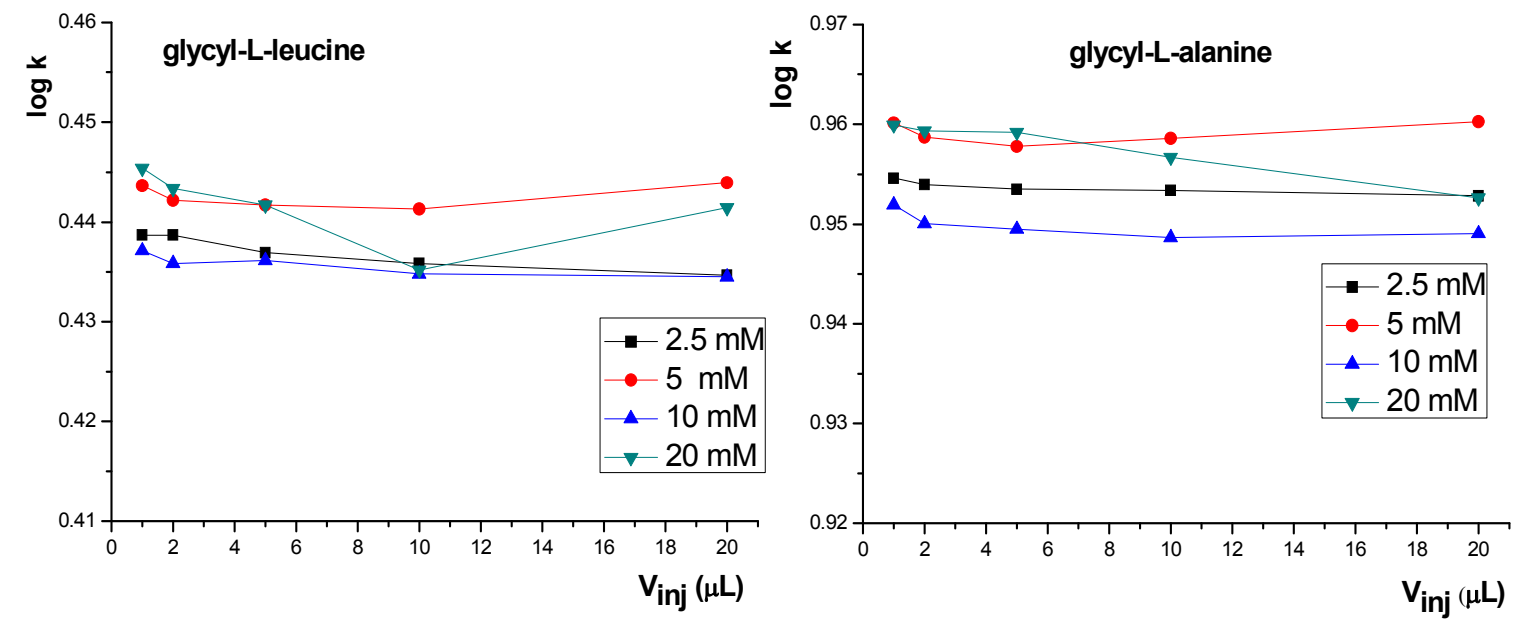

Fig. 4 - Variation of the retention factor on the injection volume and the concentration of ammonium acetate in the aqueous component (mmoles/L, denoted by $\mathrm{mM}$ ). 


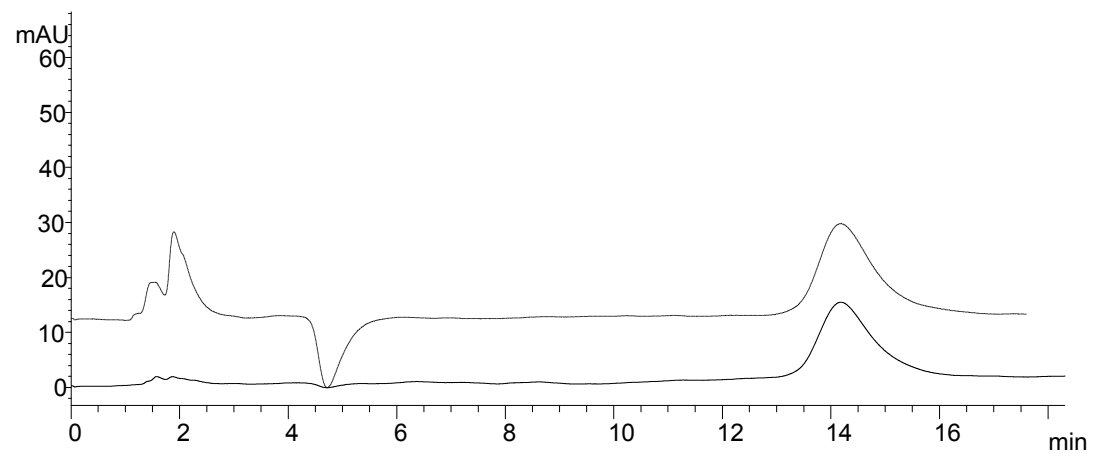

Fig. 5 - Two overlaid chromatograms resulted from the injection of 1 (continuous line) and $50 \mu \mathrm{L}$ (dashed line), respectively, of sample containing $80 \% \mathrm{ACN}$ and $20 \%$ aqueous solution with $10 \mathrm{mmoles} / \mathrm{L}$ ammonium acetate, using a mobile phase with composition: $80 \%$ ACN and $20 \%$ aqueous solution with 20 mmoles/ $\mathrm{L}$ ammonium acetate.

\section{CONCLUSIONS}

Some influences of the injected sample composition can be observed under Zic-Hilic mechanism, when injected sample volume is higher than $20 \mu \mathrm{L}$. This study showed that small variations in the composition of the injected solvent do not affect significantly the main retention parameters (retention factor, peak efficiency and peak simmetry) of the tested compounds, when the injected volume was not larger than $20 \mu \mathrm{L}$. Higher injected volumes (for example, $50 \mu \mathrm{L}$ ) can influence the retention of the less retained compound, glycyl-L-leucine, while the more retained compound is not influenced by the modification of the concentration of the ammonium acetate salt added to the sample diluent.

\section{REFERENCES}

1. M. A. Rezai, G. Famiglini and A. Cappiello, J. Chromatogr. $A$, 1996, 742, 69-78.

2. M. Hutta, M. Chalanyova, R. Halko, R. Gora, I. Rybar, M. Pajchl and S. Dokupilova, J. Sep. Sci., 2006, 229, 1977-1987.

3. S. Keunchkarian, M. Reta, L. Romero and C. Castells, J. Chromatogr. A, 2006, 1119, 20-28.

4. B. J. VanMiddlesworth and J. G. Dorsey, J. Chromatogr. A, 2012, 1236, 77-89.

5. A. Medvedovici, Va. David, Vi. David and C. Georgita, J. Liq. Chromatogr. Rel. Technolog., 2007, 30, 199-213.

6. S. Udrescu, A. Medvedovici and V. David, J. Sep. Sci., 2008, 31, 2939-2945.

7. T. Galaon, E. Bacalum, M. Cheregi and V. David, $J$. Chromatogr. Sci., 2013, 51, 166-172.
8. V. David, T. Galaon and H. Y. Aboul-Enein, J. Chromatogr. $A, \mathbf{2 0 1 4}, 1323,115-122$.

9. E. Loeser, S. Babiak and P. Drumm, J. Chromatogr. A, 2009, 1216, 3409-3412.

10. J. C. Heaton and D. V. McCalley, J. Chromatogr. A, 2016, 1427, 37-44.

11. J. Ruta, S. Rudaz, D. V. McCalley, J. L. Veuthey and D. Guillarme, J. Chromatogr. A, 2010, 1217, 8230-8240.

12. E. Bacalum, M. Tanase, M. Cheregi, H. Y. Aboul-Enein and V. David, Rev. Roum. Chim., 2016, 61, 531-539.

13. Y. Guo and S. Gaiki, J. Chromatogr. A, 2005, 1074, 71-80.

14. K. Alagesan, S.K. Khilji and D. Kolarich, Anal. Bioanal. Chem., 2017, 409, 529-538.

15. R. Zhang, D.G. Watson, L. Wang, G. D. Westrop, G. H. Coombs and T. Zhang, J. Chromatogr. A, 2014, 1362, 168-179.

16. M. Tanase, E. Bacalum and V. David. Sep. Sci. Plus, 2019, 2, 12-17.

17. I. Bohacova, R. Halko and P. Jandera, J. Sep. Sci., 2016, 39, 4732-4739.

18. Q. Zhang, F.Q. Yang, L. Ge, Y. J. Hu and Z. N. Xia, J. Sep. Sci., 2017, 40, 49-80.

19. M. R. Euerby, J. Hulse, P. Petersson, A. Vazhentsev and K. Kassam, Anal. Bioanal. Chem., 2015, 407, 91359152.

20. R. I. Chirita, C. West, S. Zubrzycki, A.L. Finaru and C. Elfakir, J. Chromatogr. A, 2011, 1218, 5939-5963.

21. Q. Wang, L. Xu and Y. W. Xue, J. Liq. Chromatogr. Rel. Technol., 2016, 39, 598-606.

22. H. Qiu, D. W. Armstrong and A. Berthod, J. Chromatogr. A, 2013, 1272, 81-89.

23. C. West and E. Auroux, J. Chromatogr. A, 2016, 1461, 92-97.

24. G. Greco and T. Letzel, J. Chromatogr. Sci., 2013, 51, 684-693.

25. L. Boulard, G. Dierkes and T. Ternes, J. Chromatogr. A, 2018, 1535, 27-43. 
